\title{
Hedgehog Inhibition Upregulates TRK Expression to Antagonize Tumor Suppression in Small Cell Lung Cancer Cells
}

\author{
HIDEYA ONISHI $^{1}$, KATSUYA NAKAMURA ${ }^{1}$, SHUNTARO NAGAI ${ }^{2}$, KOSUKE YANAI $^{1}$, \\ AKIO YAMASAKI ${ }^{1}$, MAKOTO KAWAMOTO ${ }^{1}$, AKIRA IMAIZUMI ${ }^{3}$ and TAKASHI MORISAKI ${ }^{4}$ \\ ${ }^{1}$ Departments of Cancer Therapy and Research, ${ }^{2}$ Departments of Surgery and Oncology, \\ Graduate School of Medical Sciences, Kyushu University, Fukuoka, Japan; \\ ${ }^{3}$ Shukoukai Inc., Tokyo, Japan; \\ ${ }^{4}$ Fukuoka General Cancer Clinic, Fukuoka, Japan
}

\begin{abstract}
Background/Aim: Previously we have shown that tropomyosin-related kinase B (TRKB) and Hedgehog (Hh) signalling pathways induce malignant phenotypes in many cancer types. However, results from small cell lung cancer (SCLC) clinical trials using TRK and Hh inhibitors have been disappointing. One reason for this may be the existence of crosstalk between TRKB and Hh signalling pathways. In this study, we detected negative crosstalk between the TRKB and Hh-GLII signalling pathways. Materials and Methods: The human small cell lung carcinoma cell line, SBC-5, was used. Using small interfering RNA to inhibit TRKB and $H h$ signalling, whether TRKB and Hh signaling contribute to proliferation and invasiveness in SBC-5 cells were investigated. Results: TRKB expression in GLII siRNAtransfected SBC-5 cells was higher than that of control cells. GLI1-knockdown alone did not affect invasiveness of SBC-5 cells. However, combined knockdown of TRKB and GLII significantly decreased invasiveness. Moreover, combined TRKB and GLII knockdown inhibited proliferation and migration to a greater extent than when either was inhibited alone. Conclusion: These results suggest that Hh inhibition increases TrkB expression to counter tumor suppression in $S B C-5$ cells. The combined use of TRKB and Hh inhibitors may, therefore, be useful for the treatment of refractory SCLC.
\end{abstract}

Small cell lung cancer (SCLC) is an aggressive and fastgrowing cancer, and tumors are often unresectable at the time of diagnosis. Moreover, SCLC is a refractory disease

Correspondence to: Hideya Onishi, Department of Cancer Therapy and Research, Graduate School of Medical Sciences, Kyushu University, Fukuoka 812-8502, Japan. Tel: +81 926426220, Fax: +81926426221, e-mail: ohnishi@surg1.med.kyushu-u.ac.jp

Key Words: Hedgehog signal, TrkB signal, SBC-5, small cell lung cancer. with few effective treatment options. Consequently, there is an urgent need for the development of effective therapeutic strategies for the treatment of the disease.

Tropomyosin-related kinase B (TRKB) and brain-derived neurotrophin factor (BDNF) are necessary for the normal development of the nervous system $(1,2)$. TRKB/BDNF signalling has been studied in malignant neuroendocrine tumors (NETs) such as neuroblastoma (3). We have shown that TRKB/BDNF signalling is a possible therapeutic target for large-cell neuroendocrine cancer (LCNEC) of the lung, which is a type of NET $(4,5)$. In addition, we have found that TRKB/BDNF signalling contributes to a malignant phenotype in other cancers such as lung squamous cell carcinoma and gall bladder cancer $(6,7)$. Other groups have also reported that TRKB and BDNF are associated with poor prognosis $(8,9)$. Therefore, it is possible that therapies targeting TRKB may be effective in the treatment of SCLCs of the NET type.

Hedgehog (Hh) signalling is associated with morphogenesis and is believed to cease in the adult. However, Hh signalling is re-activated in cancer and contributes to the malignant phenotype, influencing processes such as proliferation, invasion and tumorigenesis $(10,11)$. While the Hh pathway, therefore, represents a promising therapeutic target, it is known to exhibit crosstalk with other signalling pathways (12), and this crosstalk may affect the efficacy of therapies that target Hh signalling alone.

In the present study, we examined the efficacy of combination therapy that targets both TRKB and Hh to address the issue of signalling crosstalk, in an attempt to develop more effective therapeutic strategies for the treatment of SCLC.

\section{Materials and Methods}

Cell culture and reagents. Human small cell lung carcinoma cell line, SBC-5 cells were maintained in RPMI-1640 medium (Nacalai Tesque, Kyoto, Japan) supplemented with $10 \%$ fetal calf serum (FCS; Life Technologies Grand Island, NY, USA), and antibiotics (100 units/ml of penicillin and $100 \mu \mathrm{g} / \mathrm{ml}$ of streptomycin, 
A

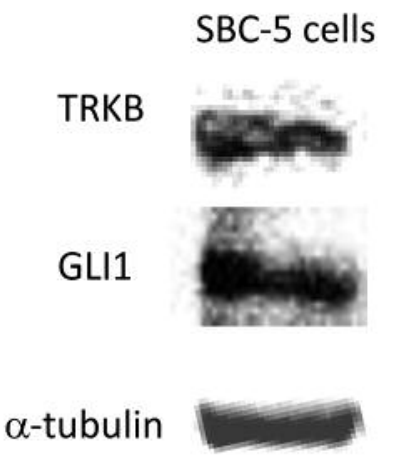
B Control si GLI1 si

Figure 1. Expressions of TRKB and GLII. A: TRKB and GLII expressions in SBC-5 cells were estimated by western blotting. B: TRKB expression in GLII siRNA transfected SBC-5 cells were investigated by western blotting.

Meijiseika, Tokyo, Japan). In proliferation assays, cell number was counted using light microscope at the indicated days.

RNA interference. Small interfering RNA (SiRNA) for Gli1 (ONTARGETplus $^{\text {TM }}$ SMART pool, L-003896), TRKB (ON-TARGETplus ${ }^{T M}$ SMART pool, L- L-003160), and negative control (ON-TARGETplus ${ }^{T M}$ Control Non-targeting siRNA, D-001810) were purchased from Dharmacon RNA Technologies (Chicago, IL, USA). Cells $\left(0.2 \times 10^{6}\right.$ cells/well) seeded in 6-well plates were transfected with $100 \mathrm{nM}$ siRNA using Lipofectamine RNAiMax Reagent (Invitrogen, Carlsbad, CA, USA) according to the manufacturer's instructions. Cells were used for experiments at 2 days after transfection.

Western blot. Whole cell extraction was performed with M-PER Reagents (Pierce Biotechnology, Rockford, IL, USA) according to the manufacturer's instructions. Protein concentration was determined with Bio-Rad Protein Assay (Bio-Rad, Hercules, CA, USA), and protein samples $(50 \mu \mathrm{g})$ were separated by electrophoresis on an SDS-polyacrylamide gel and transferred to Protran nitrocellulose membranes (Whatman GmbH, Dassel, Germany). Blots were then incubated with anti-TRKB Ab (1:200, sc-8316, Santa Cruz Biotechnology, Santa Cruz, CA, USA), antiGLI1 Ab (1:100, sc-20687, Santa Cruz Biotechnology, Santa Cruz, CA, USA), and anti- $\alpha$-tubulin Ab $(1: 1,000$, Sigma Aldrich Co., St. Louis, MO, USA) overnight at $4^{\circ} \mathrm{C}$. Blots were then incubated with the appropriate horseradish peroxidase-linked secondary antibody (Amersham Biosciences, Piscataway, NJ, USA) at room temperature for $1 \mathrm{~h}$. Immunocomplexes were detected with ECL plus Western Blotting Detection System (Amersham Biosciences, Piscataway, NJ, USA) and visualized with a Molecular Imager FX (Bio-Rad). We used $\alpha$-tubulin a protein loading control.

Invasion assay and migration assay. Cell invasion assays were performed using Matrigel-coated transwell inserts as described previously (13). In brief, the upper surface of a filter (pore size, $8.0 \mu \mathrm{m}$; BD Biosciences, Heidelberg, Germany) was coated with Matrigel basement membrane (BD Biosciences, Heidelberg, Germany). Cells were suspended in RPMI-1640 with $10 \%$ FCS. Next, we added $1.0 \times 10^{5}$ cells to the upper chamber and incubated the cells for $16 \mathrm{~h}$. After incubation, the filter was fixed and stained with Diff-Quick reagent (International Reagents, Kobe, Japan). All cells that had migrated from the upper to the lower side of the filter were counted under a light microscope (BX50; Olympus, Tokyo, Japan) at a magnification of $\times 100$. Tumor cell invasiveness was performed in triplicate wells.

Cell migration assay was performed in the same way using Matrigel-non-coated transwell inserts.

Statistical analysis. Data are presented as the means \pm standard deviation (SD). Student's $t$-tests were used to compare continuous variables between two groups. A $p$-value of $<0.05$ was considered as statistically significant.

\section{Results}

Combined knockdown of TRKB and GLII reduces proliferation in SBC-5 cells. Firstly, expression of GLI1 and TRKB was confirmed in SBC-5 cells (Figure 1A), and then the relationship between their expression was examined. Knockdown studies revealed that TRKB expression was increased in GLI1-siRNA-transfected SBC-5 cells, when compared to controls (Figure 1B). Next, the role of TRKB and GLI1 in the proliferation of SBC-5 cells was investigated. Knockdown of either TRKB or GLI1 alone reduced SBC-5 cell proliferation significantly, when compared with control cells. Combined knockdown of both TRKB and GLI1 inhibited proliferation to a greater extent than when each protein was knocked down individually (Figures $2 \mathrm{~A}$ and $\mathrm{B}$ ). These results demonstrate that co-inhibition of TRKB and Hh signallings effectively suppresses the proliferation of SCLC cells.

Combined knockdown of TRKB and GLII decreases migration and invasion in SBC-5 cells. The migratory and invasive ability of cancer cells are thought to be important determinants of tumour malignancy. Consequently, the migration and invasion of SBC-5 cells transfected with 
A
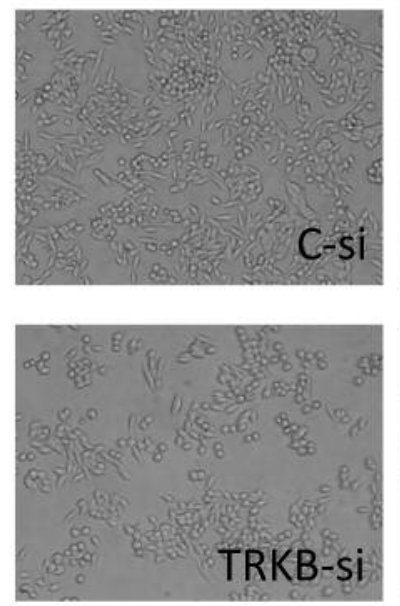
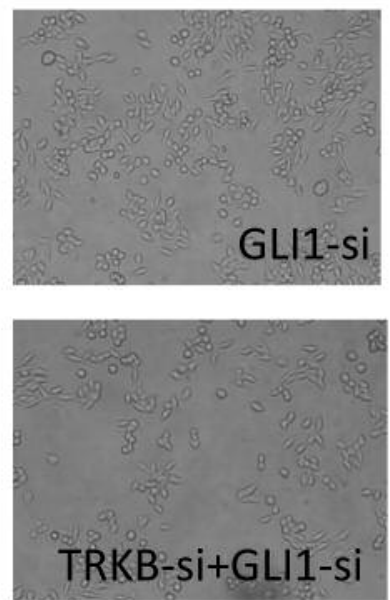

B

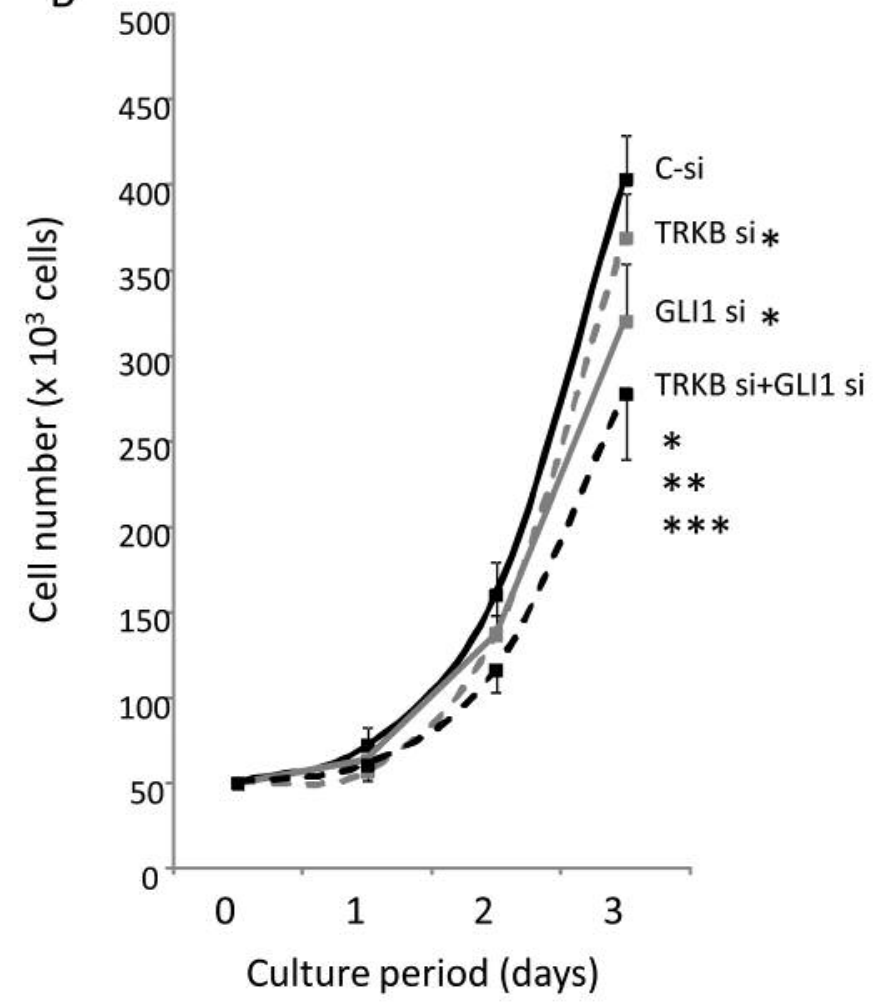

Figure 2. Proliferation in SBC-5 cells were investigated by cell counting using light microscope. A, Representative cell morphologies in GLI1 and/or TRKB inhibited SBC-5 cells on culture day 3 were shown $(\times 200)$. B, Graph shows cell number of GLII and/or TRKB inhibited SBC-5 cells. Bar shows a standard deviation. *Comparison with control siRNA group, $p<0.05 ; * *$ comparison with TRKB siRNA group, $p<0.05 ; * * *$ comparison with GLI1 siRNA group, $p<0.05$.

siRNAs targeting TRKB and/or GLI1 were analyzed. Knockdown of either TRKB or GLI1 alone reduced SBC-5 cell migration significantly, when compared to control cells. Combined knockdown of both TRKB and GLI1 decreased cell migration to a greater extent than when each target was knocked down individually (Figure 3A and B). GLI1 knockdown did not affect invasiveness in SBC-5 cells, while TRKB knockdown significantly decreased invasiveness (Figure 3C and D). Combined knockdown of TRKB and GLI1 significantly decreased invasiveness in SBC-5 cells, when compared with control and GLI1 knockdown cells (Figure 3C, D). These results demonstrate that combined inhibition of TRKB and Hh signalling effectively suppresses the migration and invasion of SCLC cells.

\section{Discussion}

In the present study, we have shown that Hh signalling inhibits TRKB expression, therefore demonstrating a negative crosstalk between these pathways (Figure 4). Yoon et al. have observed similar negative crosstalk between
GLI1 and TRKB (14). In terms of crosstalk with Hh signalling, several molecules including members of the nuclear factor-kB, oestrogen receptor- $\alpha$, and CD24/Signal transducers and activator of transcription 1 signalling pathways have been shown to regulate Sonic Hh expression (15-17). In addition, hypoxic conditions have been shown to up-regulate Smoothened transcription, while KRAS, PI3K/AKT and PKC- $\delta$ pathways have been shown to activate GLI1 (19-21). These are all examples of positive crosstalk. However, in colorectal cancer, negative crosstalk between Hh and Wnt signalling has been reported $(22,23)$. If signalling crosstalk is positive, inhibition of the target signalling pathway may also suppress signalling through the effector pathway. However, if signalling crosstalk is negative, the effect of the inhibitor may be antagonized by the activation of a previously repressed effector pathway. Clinically, if an additive or synergistic effect is not observed with some chemotherapeutic agents, then negative signalling crosstalk may exist. Given this, the results from our study suggest that an Hh inhibitor may augment TRKB expression to induce malignant phenotypes in SCLC. 
A
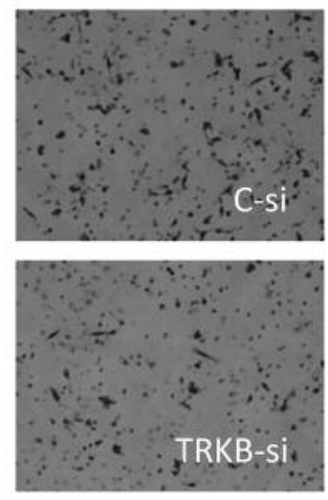

C
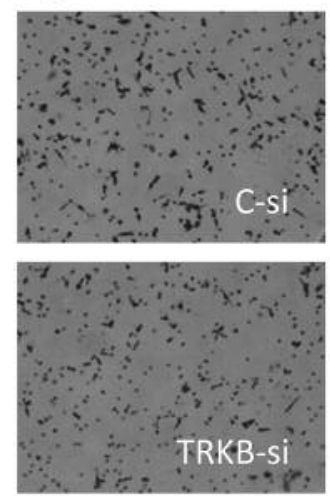
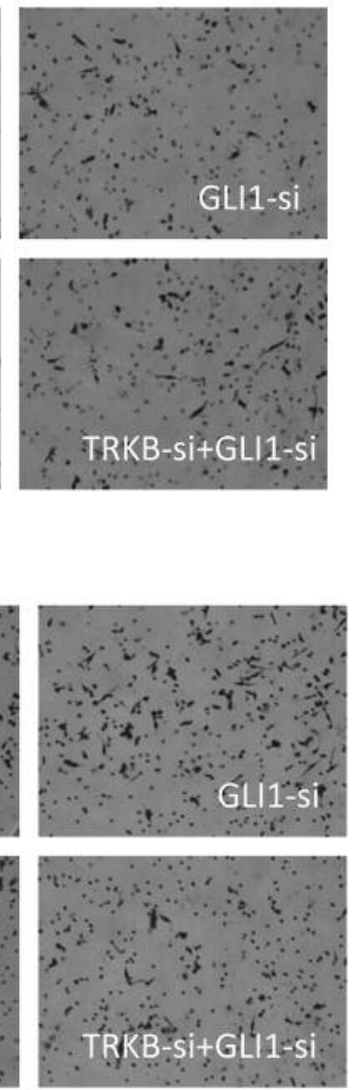

B

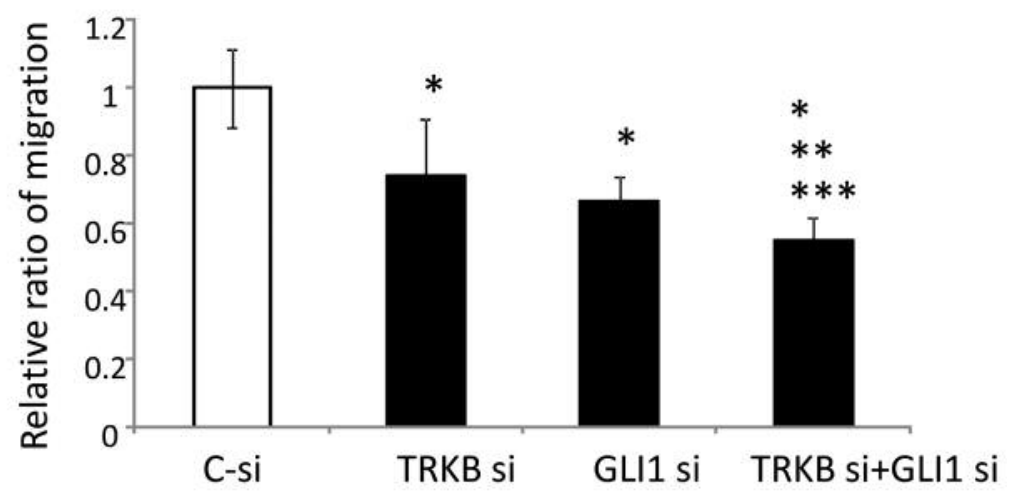

D

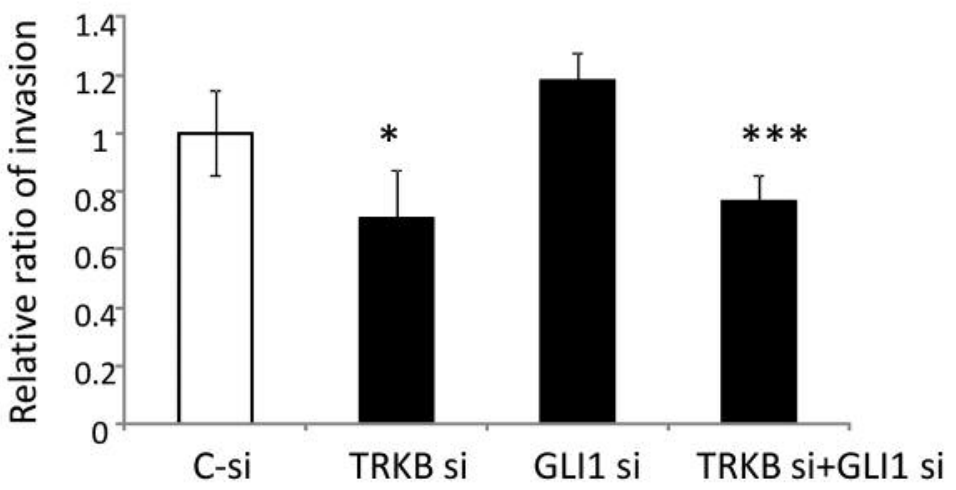

Figure 3. Cell migration and invasion were analyzed. A: Representative pictures of migrated GLI1 and/or TRKB inhibited SBC-5 cells ( $\times 200)$. B: Graph shows migrated cell number in GLII and/or TRKB inhibited SBC-5 cells. C: Representative pictures of invaded GLII and/or TRKB inhibited SBC-5 cells (×200). D: Graph shows invaded cell number in GLII and/or TRKB inhibited SBC-5 cells. Bar shows a standard deviation. *Comparison with control siRNA group, $p<0.05$; **comparison with TRKB siRNA group, $p<0.05$; ***comparison with GLII siRNA group, $p<0.05$.

Indeed, in the matrigel invasion assay, invasiveness of SBC-5 cells did not change following GLI1 knockdown (Figure 3C). This result may be a consequence of increased invasiveness driven by a mechanism associated with augmented TRKB expression, because many processes such as secretion of matrix metalloproteinases are required for invasion. However, inhibition of either TRKB or GLI1 alone led to a significant decrease in both cell proliferation and migration, and their combined inhibition resulted in an even greater decrease (Figures 2 and 3). Because rapid progression and metastasis are thought to be important characteristics of SCLC, our findings that proliferation and migration were suppressed by the combined inhibition of TRKB and GLI1 are significant.

While results from clinical trials using TRK and $\mathrm{Hh}$ inhibitors in isolation in SCLC have been disappointing, our results suggest that the combined use of Hh and TRKB inhibitors may result in an additive or synergistic effect in the clinical setting. The combinatorial use of molecular targeting agents may therefore provide a promising direction in the development of effective therapeutic strategies against refractory cancers such as SCLC.

\section{Conflicts of Interest}

The Authors declare no conflict of interest in regard to this work.

\section{Acknowledgements}

This study was supported by the Japan Society for the Promotion of Science Kakenhi Grant Number JP17K10792. The Authors thank Ms Emi Onishi for the skillful technical assistance she provided. 


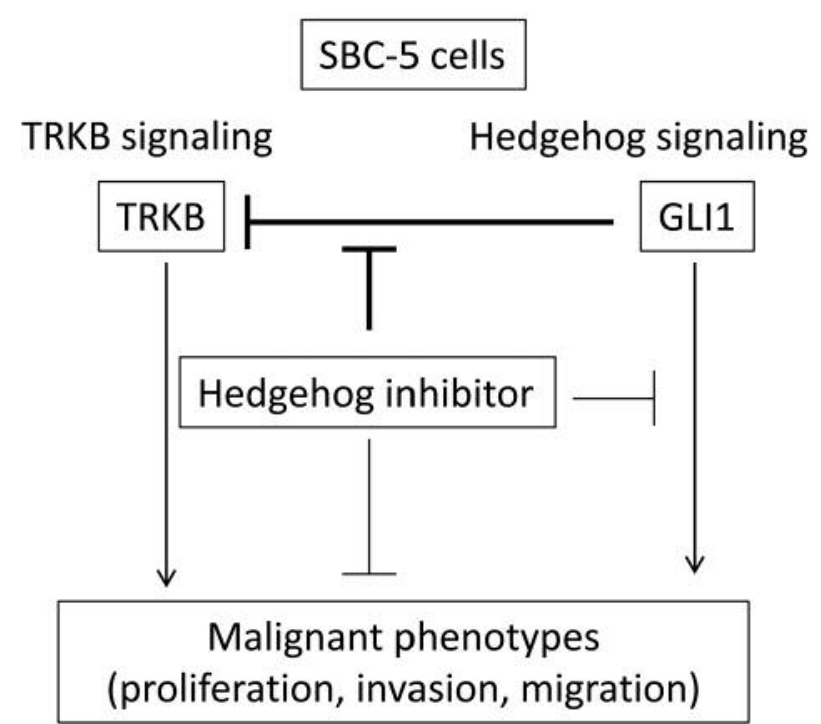

Figure 4. Schema in the present study. Negative crosstalk exists between Hh signaling and TRKB signaling. Combinational therapy using TRKB inhibitor and GLII inhibitor may be useful for small cell lung cancer. Bold lines show new results demonstrated in this study.

\section{References}

1 Klein R, Parada LF, Coulier F and Barbacid M: trkB, a novel tyrosine protein kinase receptor expressed during mouse neural development. EMBO J 8: 3701-3709, 1989.

2 Klein R, Smeyne RJ, Wurst W, Long LK, Auerbach BA, Joyner AL and Barbacid M: Targeted disruption of the trkB neurotrophin receptor gene results in nervous system lesions and neonatal death. Cell 75: 113-122, 1993.

3 Kaplan DR, Matsumoto K, Lucarelli E and Thiele CJ: Induction of TrkB by retinoic acid mediates biologic responsiveness to BDNF and differentiation of human neuroblastoma cells. Eukaryotic Signal Transduction Group. Neuron 11: 321-331, 1993.

4 Odate S, Nakamura K, Onishi H, Kojima M, Uchiyama A, Nakano K, Kato M, Tanaka M and Katano M: TrkB/BDNF signaling pathway is a potential therapeutic target for pulmonary large cell neuroendocrine carcinoma. Lung Cancer 79: 205-214, 2013.

5 Odate S, Onishi H, Nakamura K, Kojima M, Uchiyama A, Kato $\mathrm{M}$ and Katano M: Tropomyosin-related kinase B inhibitor has potential for tumor regression and relapse prevention in pulmonary large cell neuroendocrine carcinoma. Anticancer Res 33: 3699-3703, 2013

6 Ozono K, Ohishi Y, Onishi H, Nakamura K, Motoshita J, Kato M, Nakanishi R, Nakamura $\mathrm{M}$ and Oda $\mathrm{Y}$ : Brain-derived neurotrophic factor/tropomyosin-related kinase $\mathrm{B}$ signaling pathway contributes to the aggressive behavior of lung squamous cell carcinoma. Lab Invest, 2017. doi: 10.1038/ labinvest.2017.45. [Epub ahead of print]
7 Kawamoto M, Onishi H, Ozono K, Yamasaki A, Imaizumi A, Kamakura S, Nakano K, Oda Y, Sumimoto H and Nakamura M: Tropomyosin-related kinase B mediated signaling contributes to the induction of malignant phenotype of gallbladder cancer. Oncotarget 8: 36211-36224, 2017.

8 Eggert A, Grotzer MA, Ikegaki N, Zhao H, Cnaan A, Brodeur GM and Evans AE: Expression of the neurotrophin receptor TrkB is associated with unfavorable outcome in Wilms' tumor. J Clin Oncol 19: 689-696, 2001.

9 Tanaka K, Mohri Y, Nishioka J, Kobayashi M, Ohi M, Miki C, Tonouchi H, Nobori T and Kusunoki M: Neurotrophic receptor, tropomyosin-related kinase $\mathrm{B}$ as an independent prognostic marker in gastric cancer patients. J Surg Oncol 99: 307-310, 2009.

10 Onishi $\mathrm{H}$ and Katano M: Hedgehog signaling pathway as a new therapeutic target in pancreatic cancer. World J Gastroenterol 20: 2335-2342, 2014.

11 Matsushita S, Onishi H, Nakano K, Nagamatsu I, Imaizumi A, Hattori M, Oda Y, Tanaka M and Katano M: Hedgehog signaling pathway is a potential therapeutic target for gallbladder cancer. Cancer Sci 105: 272-280, 2014.

12 Onishi $\mathrm{H}$ and Katano $\mathrm{M}$ : Hedgehog signaling pathway as a therapeutic target in various types of cancer. Cancer Sci 102: 1756-1760, 2011.

13 Zhang H, Morisaki T, Matsunaga H, Sato N, Uchiyama A, Hashizume K, Nagumo F, Tadano J and Katano M: Proteinbound polysaccharide PSK inhibits tumor invasiveness by downregulation of TGF-beta1 and MMPs. Clin Exp Metastasis 18: 343-352, 2000.

14 Yoon JW, Gilbertson R, Iannaccone S, Iannaccone P and Walterhouse D: Defining a role for Sonic hedgehog pathway activation in desmoplastic medulloblastoma by identifying GLI1 target genes. Int J Cancer 124: 109-119, 2009.

15 Nakashima H, Nakamura $M$, Yamaguchi H, Yamanaka N, Akiyoshi T, Koga K, Yamaguchi K, Tsuneyoshi M, Tanaka M and Katano M: Nuclear factor-kappaB contributes to hedgehog signaling pathway activation through sonic hedgehog induction in pancreatic cancer. Cancer Res 66: 7041-7049, 2006.

16 Kameda C, Nakamura M, Tanaka H, Yamasaki A, Kubo M, Tanaka M, Onishi $\mathrm{H}$ and Katano M: Oestrogen receptor-alpha contributes to the regulation of the hedgehog signalling pathway in ERalphapositive gastric cancer. Br J Cancer 102: 738-747, 2010.

17 Suyama K, Onishi H, Imaizumi A, Shinkai K, Umebayashi M, Kubo M, Mizuuchi Y, Oda Y, Tanaka M, Nakamura M and Katano M: CD24 suppresses malignant phenotype by downregulation of SHH transcription through STAT1 inhibition in breast cancer cells. Cancer Lett 374: 44-53, 2016.

18 Onishi H, Kai M, Odate S, Iwasaki H, Morifuji Y, Ogino T, Morisaki T, Nakashima Y and Katano M: Hypoxia activates the hedgehog signaling pathway in a ligand-independent manner by upregulation of Smo transcription in pancreatic cancer. Cancer Sci 102: 1144-1150, 2011.

19 Nolan-Stevaux O, Lau J, Truitt ML, Chu GC, Hebrok M, Fernández-Zapico ME and Hanahan D: GLI1 is regulated through Smoothened-independent mechanisms in neoplastic pancreatic ducts and mediates PDAC cell survival and transformation. Genes Dev 23: 24-36, 2009.

20 Riobó NA, Lu K, Ai X, Haines GM and Emerson CP Jr.: Phosphoinositide 3-kinase and Akt are essential for Sonic Hedgehog signaling. Proc Natl Acad Sci USA 103: 4505-10, 2006. 
21 Riobo NA, Lu K and Emerson CP Jr.: Hedgehog signal transduction: signal integration and cross talk in development and cancer. Cell Cycle 5: 1612-1615, 2006.

22 Akiyoshi T, Nakamura M, Koga K, Nakashima H, Yao T, Tsuneyoshi M, Tanaka M and Katano M: Gli1, down-regulated in colorectal cancers, inhibits proliferation of colon cancer cells involving Wnt signalling activation. Gut 55: 991-999, 2006.
23 Yanai K, Nakamura M, Akiyoshi T, Nagai S, Wada J, Koga K, Noshiro H, Nagai E, Tsuneyoshi M, Tanaka M and Katano M: Crosstalk of hedgehog and Wnt pathways in gastric cancer. Cancer Lett 263: 145-156, 2008.

Received July 4, 2017 Revised July 19, 2017 Accepted July 20, 2017 